\title{
Encéphalite à anticorps anti-NMDAR révélée par une symptomatologie psychiatrique
}

\author{
Anti-NMDAR Encephalitis: Psychiatric Presentation of a Neurological Disease
}

\author{
F. Balen $\cdot$ L. Moreno $\cdot$ K. Daros Oudrhiri \\ Reçu le 26 septembre 2018; accepté le 27 novembre 2018 \\ (C) SFMU et Lavoisier SAS 2018
}

\section{Introduction}

L'encéphalite à anticorps anti-N-Methyl-D-Aspartate Receptor (NMDAR) est l'encéphalite auto-immune la plus fréquente de ce groupe nosologique rare. Sa présentation clinique classique chez le jeune adulte étant d'allure psychiatrique, l'erreur dans le diagnostic initial est fréquente. Cette présentation pose la question des liens entre psychiatrie et substrat organique. Nous rapportons ici le cas d'une patiente qui se révèle atteinte d'une encéphalite auto-immune à antiNMDAR de mécanisme paranéoplasique en rapport avec un tératome du médiastin.

\section{Observation}

Une patiente de 23 ans, sans antécédents notables, se présente aux urgences accompagnée d'une amie. Elle présente depuis la vieille au soir une angoisse, un comportement décrit comme « étrange » par son amie. La patiente dit se sentir « sortie de la réalité », ne reconnait pas le clinicien qui l'a examinée initialement lorsqu'il vient la réévaluer, mais « reconnaît sa voix ». Il n'y a pas de notion de perte de contact ni de mouvements anormaux. L'élément déclenchant semble être la reprise du travail qui devait avoir lieu le jour de son admission : la patiente était en arrêt maladie depuis un mois pour surmenage dans le cadre d'une activité dans la restauration rapide. L'examen clinique, en particulier neurologique, est sans anomalie : apyrexie, pas de céphalée ni de

F. Balen $(\bowtie) \cdot$ L. Moreno $\cdot$ K. Daros Oudrhiri

Pôle de médecine d'urgence, CHU de Toulouse, place du Docteur-Baylac, TSA 40031,

F-31059 Toulouse cedex 09, France

e-mail : balen.f@chu-toulouse.fr

F. Balen

Inserm, UMR 1087, université Toulouse-III,

F-31000 Toulouse, France raideur méningée. La TDM cérébrale sans produit de contraste est sans particularité. Le bilan biologique ne retrouve pas d'anomalie, les toxiques urinaires négatifs, et l'EEG réalisé pour ne pas méconnaître une épilepsie temporale est rendu normal. La patiente est orientée en psychiatrie pour « burn-out».

Au cinquième jour d'hospitalisation en psychiatrie, l'état de la patiente se dégrade : décrite comme catatonie atypique, elle est retransférée aux urgences. Elle y est retrouvée somnolente, réactive à la douleur, mutique et présentant plusieurs mouvements stéréotypés de la mâchoire à type de mâchonnements. Un nouvel EEG est réalisé retrouvant un tracé ample et ralenti, sans activité épileptique, mais un aspect en delta-brush. L'IRM cérébrale retrouve un discret hypersignal hippocampique et amygdalien bilatéral en FLAIR. Le bilan biologique sanguin reste sans particularité, et les premiers résultats de la ponction lombaire rapportent une hypercellularité (50 éléments nucléés) avec protéinorachie et glycorachie normales. L'aggravation de son état de vigilance menant à un coma aréactif conduit à une intubation et un transfert en service de réanimation.

Le diagnostic d'encéphalite auto-immune est alors évoqué devant ce tableau pseudopsychiatrique de novo évoluant vers la catatonie avec stéréotypies orofaciales puis le coma, associé à des anomalies en delta-brush à l'EEG. Le dosage d'anticorps anti-NMDAR dans le LCR est rendu positif dans un second temps. Un traitement par corticoïdes et immunoglobulines à forte dose est alors entrepris. La TDM-TAP, à la recherche d'une étiologie néoplasique à cette encéphalite, retrouve une masse médiastinale de $6 \times$ $3 \times 7 \mathrm{~cm}$, dont l'anatomopathologie après exérèse chirurgicale s'avère être celle d'un tératome.

Après de nombreux mois de réanimation, la malade sera transférée trachéotomisée en service de rééducation où son rétablissement est lent : le score MIF (Mesure de l'indépendance fonctionnelle) à un an de sa consultation initiale est à 107/126, du fait de persistance de troubles des interactions sociales, mnésiques et dysexécutifs. 


\section{Discussion}

L'encéphalite auto-immune est une pathologie cérébrale rare, d'une incidence estimée de 5 à 8 cas/100 000personnes par an [1]. Le principal mécanisme décrit des encéphalites auto-immunes est l'auto-immunité médiée par les anticorps anti-NMDAR (N-Methyl-D-Aspartate Receptor : récepteur de surface des neurones) [1,2]. Le facteur déclenchant des encéphalites auto-immunes semble être viral [3] ou néoplasique [4]. Parmi les encéphalites à anti-NMDAR en particulier, l'association avec un tératome ovarien semble fréquente [5].

Avec un âge médian de survenue de 21 ans, l'encéphalite auto-immune à anti-NMDAR est une pathologie du jeune adulte, à prédominance féminine (sex-ratio de $4: 1$ ) [6].

La symptomatologie décrite initialement revêt un aspect psychiatrique d'apparition rapidement progressive chez des patients sans antécédents de cette nature : insomnies, irritabilité, changement de comportement, tristesse ; et conduit souvent à une hospitalisation en psychiatrie. La progression se fait dans les jours ou semaines, vers des troubles de la mémoire ou un mutisme. Les dyskinésies orofaciales, crises d'épilepsie (présente dans $75 \%$ des cas [7]), altération de la vigilance d'intensité variable et apnées centrales [8] doivent faire proposer des explorations complémentaires. L'IRM cérébrale peut retrouver des hypersignaux en séquence FLAIR, non systématisés, corticaux ou sous-corticaux, présents dans seulement $30 \%$ des cas [6]. L'EEG peut retrouver des anomalies caractéristiques en «delta-brush» [9]. Le diagnostic d'encéphalite à anti-NMDAR est confirmé par la détection dans le LCR d'anticorps dirigés contre l'épitope GluN1 du récepteur NMDA, la détection de cet anticorps dans le sérum étant moins sensible (14\% de faux négatifs) [10].

Le traitement repose sur l'ablation précoce de la néoplasie d'une part, et sur l'utilisation de corticoïdes et d'immunoglobulines à forte dose d'autre part [1]. La vitesse de guérison est lente : $81 \%$ des malades n'ont plus ou peu de symptômes à deux ans [6].

En conclusion,les encéphalites auto-immunes et leur présentation psychiatrique sont un piège diagnostique intéressant à connaître. Elles posent également la question d'un probable substrat organique encore mal connu aux patholo- gies psychiatriques : des autoanticorps anti-NMDAR ayant été retrouvés chez certains patients schizophrènes [11]. Les psychoses seraient-elles des pathologies auto-immunes atteignant les récepteurs NMDA et GABA des surfaces neuronales [12] ?

Conflits d'intérêts : les auteurs déclarent ne pas avoir de liens d'intérêts.

\section{Références}

1. Dalmau J, Graus F (2018) Antibody-mediated encephalitis. N Engl J Med 378:840-51

2. Harutyunyan G, Hauer L, Dunser MW, et al (2017) Autoimmune encephalitis at the neurological intensive care unit: etiologies, reasons for admission and survival. Neurocrit Care 27:82-9

3. Armangue T, Moris G, Cantarin-Extremera V, et al (2015) Autoimmune post-herpes simplex encephalitis of adults and teenagers. Neurology 85:1736-43

4. Lancaster E, Lai M, Peng X, et al (2010) Antibodies to the GABA(B) receptor in limbic encephalitis with seizures: case series and characterisation of the antigen. Lancet Neurol 9:67-76

5. Acien P, Acien M, Ruiz-Macia E, Martin-Estefania C (2014) Ovarian teratoma-associated anti-NMDAR encephalitis: a systematic review of reported cases. Orphanet J Rare Dis 9:157

6. Titulaer MJ, McCracken L, Gabilondo I, et al (2013) Treatment and prognostic factors for long-term outcome in patients with anti-NMDA receptor encephalitis: an observational cohort study. Lancet Neurol 12:157-65

7. Grinea A, Demeret S, Bolgert F, et al (2017) Une jeune femme avec des symptômes psychiatriques subaigus aux urgences. Rev Prat 67:641-4

8. de Montmollin E, Demeret S, Brule N, et al (2017) AntiN-Methyl-D-Aspartate Receptor encephalitis in adult patients requiring intensive care. Am J Respir Crit Care Med 195:491-9

9. Schmitt SE, Pargeon K, Frechette ES, et al (2012) Extreme delta brush: a unique EEG pattern in adults with anti-NMDA receptor encephalitis. Neurology 79:1094-100

10. Gresa-Arribas N, Titulaer MJ, Torrents A, et al (2014) Antibody titres at diagnosis and during follow-up of anti-NMDA receptor encephalitis: a retrospective study. Lancet Neurol 13:167-77

11. Le Guen E, Doukhan R, Hamdani N, et al (2015) Anti-NR1 antibodies in anti-N-Methyl-D-Aspartate receptor encephalitis and schizophrenia. Med Sci 31:60-7

12. Al-Diwani AAJ, Pollak TA, Irani SR, Lennox BR (2017) Psychosis: an autoimmune disease? Immunology 152:388-401 